\title{
The PEPL system for control of experiments by a PDP-8 computer
}

\author{
DAVID J. GETTY \\ Brown University, Providence, Rhode Island 02912
}

\begin{abstract}
PEPL (Psychology Experiment Programming Language) is a real-time, on-line experimental control system designed for a 4K PDP-8 computer with minimal additional hardware. The system, consisting of the PEPL language, a symbol table for standard DEC assemblers, and an operating system, is capable of asynchronous, timed control of multiple subjects or experiments. The language offers the user high-level instructions for execution control, computation, timing, and I/O control.
\end{abstract}

The development of the PEPL (Psychology Fxperiment Programming Language) system has followed an evolutionary path. For several years, we have used earlier versions of this system to control experiments on human sensory information processing, using a $4 \mathrm{~K}$ DEC PDP-8 minicomputer. Throughout the development, we have been motivated (a) by the need for a high-level language which permits rapid. intuitive programming of experimental procedures, and which does not require a special compiler, and (b) by the need for an operating system that can control experiments utilizing a 4K PDP-8 family minicomputer with minimal additional hatrdware.

From these general requirements, a number of more specitic design criteria have emerged:

(1) The system should allow the simultaneous. asynchromous control of multiple experiments for multiple subjects within an experiment). Since the amount of memory available to the user is presumed to be minimal, it should be possible to share one copy of a procedure among several subjects running in the sime experiment.

(2) The system should handle all details of the management of $1 / 0$ with experiment and system devices.

(3) Since many behavioral experiments involve interactive procedures, the system should allow the user to carry out on-line data processing and logical lesting.

(4) The system should be able to provide the current time and to eoncurrently time-out a number of independent, overlapping time intervals.

The development of PEPL is the result of contributions from the Psychology 181 class (Fall, 1973) on Computer Applications in Psychology, and the major efforts of Paul Anagnostopoulos, John Crawford, Joan Diaz, Ken Hoadley, and Greg Wood. Special thanks are due Ken Hoadley for his dedication to the project and his critical reading of this paper. Requests for reprints should be sent to David J. Getty. Department of Psychology, Brown University, Providence, Rhode Island 02912. It is anticipated that the PEPL system (operating systen source tape, system symbol tabie, and user's manual) will be made availible through DECUS.
(5) The design of the operating system should be as modular as possible to permit addition, deletion, or modification of routines in the operating system.

The ways in which these criteria have been met in PIIL will be discussed in the remainder of the paper.

\section{EVENTS AND TASKS}

The structure of the PEPL language and operating system is based on events and event processing tasks. An experiment may be viewed as an interactive seyuence of events, each occurring at a defined moment in lime under the control of either the experimenter, as specified by the experimental procedure. or the subject.

In writing a program to control an experiment, the user crealtes at set of event processing tasks, each associated with one of the possible events in the experiment. There are two types of functions served b) talsks. All tasks contain instructions to carry out "hatever processing is required by the occurrence of the initiating event. In addition, some tasks also contain instructions which determine characteristics, such as identity or time of occurrence, of future events in the experiment. Examples of tasks may be found in lhe sample program discussed later in the paper.

\section{TIMESHARING}

The system is designed to allow a number of experimental procedures, referred to as jobs, to be controlled simultaneously and asynchronously. The procedures may be totally unrelated to one another or may be identical, as when several subjects are run in the same experiment. In the latter case, only a single copy of the procedure object code need be held in memory. This is an important consideration since one of the severe timesharing constraints in the system is the limited amount of memory available for the procedure code. The other major timesharing comstraint is the accuracy of system timing. As more fobs atre timeshared. deliy's in the processing of events 


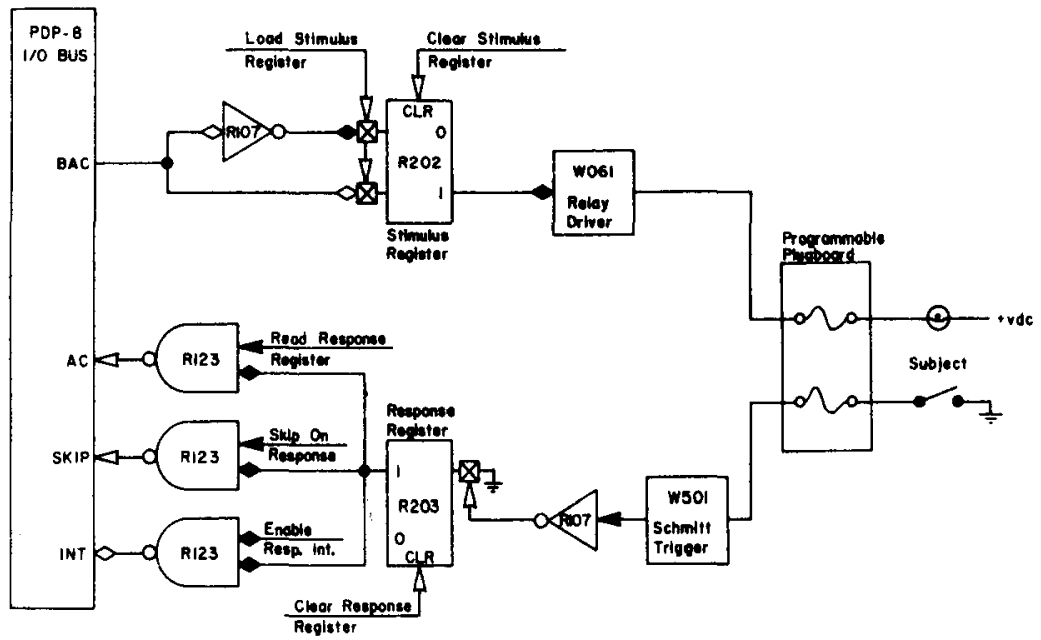

Figure 1. Experiment interface logic diagram, showing a single representative line in the stimulus register and a single representative line in the response register.

by the operating system may eventually degrade the accuracy of the system timing routines. The point at "hich this occurs will depend on the timing accuracy required. the period of the timebase, and the load on lle system.

During the execution of a task, the identity of the job in control, its job number. is maintained by the operating system and made available to the user as an integer variable named JOB. If future events are enabled or scheduled by the user from within a task. the system automatically appends the current job number to its internal record of scheduled events. When these events occur in the future, they are identilied with the correct job number.

Requests for service from system and experimental devices are detected by the computer interrupt circuit which is turned on whenever the operating system monitor is in control and turned off whenever control is passed to a user task. This method removes some of the timing delays that result when device polling is used instead. Service priorities are established by the position of device test instructions in the interrupt skip chain.

\section{HARDWARE}

\section{Minimal Configuration}

The PEPL operating system requires the following minimal hardware configuration: (1) DEC PDP-8 lamily computer with $4 \mathrm{~K}$ memory. (2) Teletype (for operator communication). (3) High-speed paper-tape reader/punch (or other data storage device). (4) Programmable real-time clock. (5) External stimulus register (controlling up to 12 binary-valued stimulus lines per register). (6) External response register (controlling up to 12 binary-valued response lines per register).

The operating system was written using the harduare instruction set of the PDP-8/S computer. It is therefore expected to be upward compatible with all wh the more recent nembers of the PDP- 8 family. The current version was designed specifically to operate in a $4 \mathrm{~K}$ memory. No specitic provision was made for taking advantage of additional memory. We anticipate that a future version of the system will be written to elfectively utilize additional memory tields.

The current system assumes paper tape to be the primary medium for data input and output. An installation having another data storage device such as matglietic tape. Dectape, or disk could replace the paper-tape handlers in the operating system with handlers for one of these other devices.

\section{Experiment Interface}

The system assumes the existence of both a hardware stimulus (output) register to control binary-valued lines into the experimental environment and a hardware response (input) register to store the occurrence of switch closures on a number of response lines coming fon the experimental environment. The actual hardware implementation of these two registers can take many forms. As an illustration, the implementation in my laboratory is described below and diagrammed in Figure 1. The tigure shows the circuitry. using negative logic modules, for a single representative bit in each of the two registers. The stimulus register acts as an external memory for the state of the stimulus lines, loaded by jam-transfer from the accumulator. Each thip-llop in the register controls a relay driver which supplies current to drive stimulus lights. relays, etc. Experimental devices are wired to the receiver of a removable plugboard rather than to the stimulus lines themselves so that a snall number of lines can, at different times, serve a large number of devices. Thus, the assignment of stimulus and response lines to particular experimental devices can be changed by simply inserting another plugboard.

Response lines are contigured to detect contact closures to ground. The closure is filtered for bounce and shaped by a Schmitt trigger to set a flip-flop in the response register. A response on any of the 
response lines will cause an interrupt if the interrupt bus gates and the computer interrupt circuit are all enabled. A response can also be detected by a skip-on-response IOT instruction, which enables the skip bus gate. When a response is detected, the contents of the response register are read into the accumulator with a read-response-register IOT instruction.

There are no logical constraints on the number of stimulus or response lines that can be controlled by the operating system. The routines currently in the operating system are written for a single stimulus and a single response register, each of 12 or fewer bits; however, somewhat longer routines to control multiple registers also exist.

Other types of devices used by a particular installation (such as $A / D$ and $D / A$ converters, subject terminals, random-access projectors, etc.) can be brought under control of the operating system without hardware moditication by adding device handling routines to the operating system. These routines are then made available to the user by adding appropriate instructions to the PEPL language. This requires only that the mnemonics and machine code translations (usually a subroutine call) be added to the PEPL symbol table used by the assembler.

\section{SOFTWARE}

The procedure for writing and running a program to control a specitic experimental procedure involves three steps: (a) writing a program in the PEPL language, consisting of an appropriate set of tasks; (b) assembling the source code into object code, using any standard DEC assembler, supplied with the PEPL table; and (c) loading the object code of both the experimental procedure and the operating system into memory for execution.

The PEPL system differs from some other PDP-8 based control systenı (Kadden, 1974; Polson, 1973; Schneider \& Scholz. 1973; Wallsten, 1972) in that it uses an assembler to produce the object code rather than a specially designed compiler. This has not prevented the inclusion of relatively high-level instructions. While it is true that the syntax necessitated by an assembler is not as natural as that possible with a compiler. there are several offsetting advantages of the assembler. First, the user can include assembly language subroutines directly in his program without difficulty. Second, the transportability of the system is increased since all PDP-8 systems come equipped with some version of the DEC PAL assembler appropriate to the system hardware. A compiler, on the other hand, would have to be modilied at each installation to operate with the local system configuration. Finally, the set of PEPL mnemonics can be altered as desired by the user by changing the system symbol table.

\section{The PEPL Language}

The set of instructions in the PEPL language fall into four categories: (a) computational, (b) execution control. (c) timing, and (d) input/output. Each inslruction consists of a defining mnemonic followed by a list of arguments. Arguments are generally the names of integer, floating point, or character string variables. However, specific arguments in several instructions may be either variable names or numerical values. For the most part, mnemonics are translated by the assembler into subroutine calls to the operating system. The design of the system is such that the user never need by concerned with the direct paige addressing constraints of the PDP-8, regardless of program length. Whenever possible, instruction arguments have been ordered from left to right to agree with their serial order in the logical flow of processing.

\section{Computational Instructions}

Numerical variables. Numbers may be represented in the system either as double precision integer variables or as floating point variables. The standard dyadic arithmetic operations are available. For example, the instruction "MULT ; I1;I2;I3" multiplies the integer variable 11 by the integer variable 12 and places the result in $\mathbf{3}$. Corresponding operations exist for lloating point variables, the instruction mnemonics being suffixed with the letter F (e.g., MULTF). Instructions are available to transform floating point numbers into integers, either by truncation or rounding, and vice versa. Instructions exist to set variables to numerical values, copy variables, negate variables, and, in the case of integer variables, to increment or decrement variables by 1 .

The system allows the creation and manipulation of onc-dimensional tables through instructions to get or store a variable as the ith entry in a named table. For example, the instruction "TGETF ;LIST; $I ; X$ " gets the ith entry in a table of floating point numbers called LIST and assigns it to the variable named $X$. Tables can also be initialized to a specific value.

The computational routines also include a statistically acceptable pseudorandom number generator. It generates floating point numbers uniformly distributed between limits which can be set by the SCALEF instruction. These limits default to the unit interval. The seed value for the routine can be set and retrieved by the user.

Character string variables. Character strings can be manipulated with the TPUTC and TGETC insuructions. They enable the user to embed, conciatenate, or extract a substring of characters of variable length from a character table. For example, the instruction "TPUTC ;PHRASE; I;WORD;5" places a tive-character substring named WORD into a character string named PHRASE, starting at its ith 
clement. Chamacter strings can also be compared using the logical IFC instruction.

\section{Execution Control Instructions}

Conditional execution of program segments is possible using a logical IF instruction of the form "IF ; I1;OP;I2;LABL", where OP is one of the set of ligical operators GE, LE, GT, LT, EQ, and NE. If the condition is true, execution branches to LABL. Logical tests are also available for floating point variables (IFF) and for character strings (IFC) of any length. Unconditional transfer of control is specified by the GOTO instruction.

Looping through a set of instructions is possible using a DO instruction. The starting, ending, and increment values of the index variable may be either integer variables or numeric values.

Error conditions can be signaled either by the WARNING instruction, which prints a numbered warning message on the operator Teletype and continues execution, or by the ERROR instruction, which prints a numbered error message and halts execution of the job presently in control.

Tasks usually end with an ENDTASK instruction which returns control to the operating system.

\section{Timing Instructions}

The operating system contains a sophisticated set of timing routines, based in part on software written by Wickens, Howard, Rice, and Millward (1972) for GASPS. The software, utilizing a single 12-bit programmable hardware clock, is able to keep track of the current time and to act as an interval timer for a number of overlapping time intervals. Time intervals are represented as double precision integers, with the unit of time detined as the period of the hardware clock timebase. The overhead in handling the hardware clock is small compared to that in some systems, since an interrupt occurs only when the 12-bit hardware register overflows rather than on every pulse from the timebase. The current time can be obtained by the CLOCK instruction. The user can request that a specitic event occur at a future time by issuing the instruction "TIMEOUT ;INT;EVENT", where INT is the name of the interval to be timed out and EVENT is the name of the task that is to be executed at the end of the interval. An interval that is currently being timed out can be canceled by the "CANCEL ;EVENT;JOB" instruction. In addition, the timing of all intervals associated with the job currently in control can be terminated by "CANCEL ;ALL;JOB".

\section{Input/Output Instructions}

Stimulus register. The stimulus lines are controlled by the instructions TURNON and TURNOFF. Convenient mnemonics for devices are possible by detining them to the assembler; e.g., "TURNON ;LIGHT" would activate Stimulus Line 3 if the definition "LIGHT $=3$ " were included in the program.

Response register. Instructions for the response register control whether or not a particular response line is conbled, detine the job to which it is assigned, and specify the task to be executed when the response occurs. The instruction "ENABLE ;SWITCH; EVENT" would enable the response line defined by SWITCH, assign it to the job that is currently executing, and specify EVENT as the task to be exccuted when the response occurs. The instruction "DISABLE ;SWITCH" would cause future interrupts produced by the SWITCH response to be ignored in effect by the operating system.

ASCII devices. The input and output of decimal numbers or character strings to ASCII-coded devices is controlled by the READ and WRITE instructions. As an example, the instruction "READ ; HSR;F;3; NUM1;NUM2;NUM3" specifies that three floating point numbers be read from the high-speed reader and placed in NUM1, NUM2, and NUM3. A11 numerical input is free-form. Another format type exists to specify that $\mathrm{N}$ numbers be read into a named table, where $N$ is either an integer variable or a numerical value. Both list and table input formats exist for integers as well.

Two other input formats specify that a string of $\mathrm{N}$ characters be read into a table, stored either as full 8-bit codes, one character per location, or as stripped 6-bit codes, packed two characters per location. Comversion of a character string stored in memory to one or more numerical values can be accomplished by a core READ instruction. For example, "READ ; CHARST;D;2;NUM1;NUM2" would read characters serially from a string named CHARST until conversion of two integer numbers was completed. These two numbers would be placed in NUM1 and NUM2.

Corresponding output formats exist for the WRITE statements with additional arguments included to specify field widths for numerical output. For example, "WRITE ;TTY;FT;3;5;2;DATA" specifies that three floating point numbers stored in the table DATA be printed on the Teletype with a total field width of five places and with two places reserved after the decimal point. Formats also exist to insert carriage return/line feeds and spaces into the output.

\section{A SAMPLE PROGRAM}

As an example of progranming with the PEPL language, the fixed-set procedure for Sternberg's (1966) item-recognition experiment is shown in Table 1. The procedure consists of seven tasks. The list of variables and constants which would normally follow the tasks, required by the assembler to assign locations, has been omitted for brevity. All numbers entered in a user program are decimal values. 
Table 1

Sample Program: Sternberg's Fixed-Set Item Recognition Paradigm

\begin{tabular}{cl}
\hline /Initialization Task & \\
READ & ; HSR ; D ; 5 ; NTRIAL ; NPOS ; NNEG ; ITI ; FOREP \\
READ & ; HSR ; C ; NPOS ; POSSET \\
READ & ; HSR ; C ;NNEG ; NEGSET \\
SET & ; TRIAL ; 1 \\
FLOAT & ; NPOS ; FPOS \\
FLOAT & ; NNEG ; FNEG \\
GOTO & ; NXTRL
\end{tabular}

/Task NXTRL: Set up for next trial

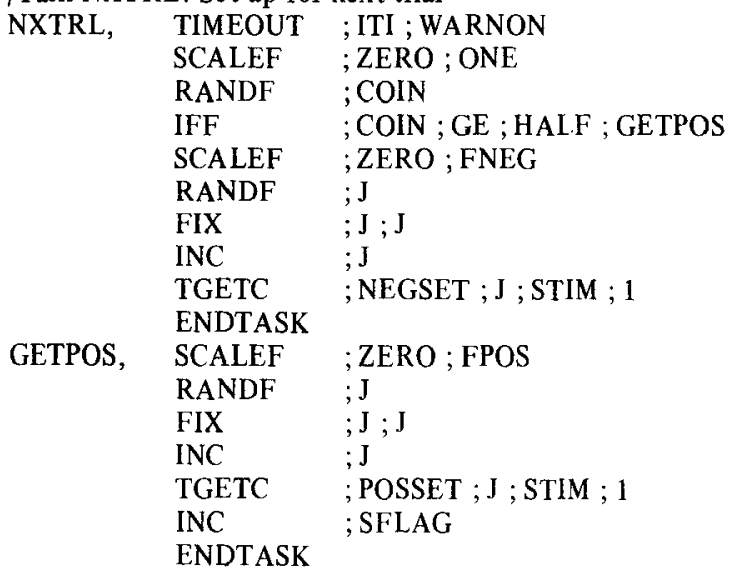

/Task WARNON: Turn on warning light

WARNON, TURNON ; LIGHT

$\begin{array}{ll}\text { TIMEOUT } & \text {; FLASH ; WARNOF } \\ \text { TIMEOUT } & \text {; FOREP ; STIMON }\end{array}$

ENDTASK

/Task WARNOF: Turn off warning light

WARNOF, TURNOFF ; LIGHT ENDTASK

/Task STIMON: Present stimulus digit to subject

$\begin{array}{lll}\text { STIMON, } & \text { WRITE } & \text {;CRT ; C ; } 1 \text { STIM } \\ & \text { CLOCK } & \text {; TIME1 } \\ \text { ENABLE } & \text {; KEYP ; RESPP } \\ \text { ENABLE } & \text {; KEYN ; RESPN } \\ \text { ENDTASK } & \end{array}$

/Task RESP: Process subject response and test for new trial

\begin{tabular}{|c|c|c|}
\hline \multirow{9}{*}{$\begin{array}{l}\text { RESPP, } \\
\text { RESPN, }\end{array}$} & & \\
\hline & INC & ; RFLAG \\
\hline & CLOCK & ; TIME2 2 \\
\hline & WRITE & ;CRT ; C ; 1 ; ERASE \\
\hline & DISABLE & ; KEYP \\
\hline & DISABLE & ;KEYN \\
\hline & IF & ;RFLAG ; LQ ; SFLAG ; CRESP \\
\hline & WRITE & ;CRT ; T ; 5 ;ERR \\
\hline & GOTO & ; TIMEFB \\
\hline \multirow{11}{*}{$\begin{array}{l}\text { CRESP, } \\
\text { TIMEFB, }\end{array}$} & WRITE & ;CRT ; T ; 7 ;CORR \\
\hline & TIMEOUT & ; FEEDBK ; FEEDOF \\
\hline & SUB & ; TIME2 ; TIME1 ; RT \\
\hline & WRITE & ; HSP ; C ; 1 ; STIM \\
\hline & WRITE & ; HSP ; D ; $3 ; 6$; RFLAG ; RT ;CRLF \\
\hline & SET & ; SFLAG ; 0 \\
\hline & SET & ; RFLAG ; 0 \\
\hline & $\mathrm{INC}$ & ; TRIAL \\
\hline & IF & ; TRIAL ; LE ; NTRIAL ; NXTRL \\
\hline & WRITE & ;CRT ; T ; $17 ;$ ENDEXP \\
\hline & ENDTASK & \\
\hline
\end{tabular}

/Task FEEDOF: Erase feedback message from CRT

FEEDOF, WRITE ;CRT; $\mathrm{C} ; 1$; ERASE
/Read parameters

/Read set of positive stimulus digits

/Read set of negative stimulus digits

/Set TRIAL=1

/FPOS=Float(NPOS)

/FNEG=Float(NNEG)

/Jump to task NXTRL

/Begin timing out intertrial interval /Scale RN generator to $(0,1)$

$/$ Fetch random number

$/$ If COIN $\geqslant 0.5$, go to GETPOS

/Scale RN generator to ( 0, FNEG)

/Fetch random number

/Truncate $\mathrm{J}$ to integer

/ Increment $J$ so that $1 \leqslant J \leqslant F N E G$

$/$ STIM=NEGSET $(\mathrm{J})$

/Return control to op. sys.

Scale RN generator to (0, FPOS)

$/ F e t c h$ random number

/Truncate $J$ to integer

/Increment $J$ so that $1 \leqslant J \leqslant$ FPOS

/STIM=POSSET(J)

/Set pos. stim. flag

/Return control to op. sys.

/Turn on warning light

/Time out flash duration

/Time out foreperiod

/ Return control to op. sys.

/Turn off warning light

/ Return control to op. sys.

/Display digit on subject CRT

$/$ Get and store current time

/Enable positive response key

/Enable negative response key

/Return control to op. sys.

/Positive resp. entry; set flag

/Neg. resp. entry; get current time

/Erase subject CRT screen

/Disable positive response key

/Disable negative response key

/RFLAG=SFLAG?

/No. Write "ERROR" on CRT

/Yes. Write "CORRECT" on CRT

/Timeout feedback message

/ Reaction time=TIME2-TIME 1

$/$ Record stimulus digit presented

/Punch response type, RT, \& CR/LF

/Reset SFLAG to 0

/ Reset RFLAG to 0

$/$ TRIAL $=$ TRIAL +1

/If TRIAL $\leqslant$ NTRIAL, go to NXTRL

/Write "END OF EXPERIMENT" on CRT

/Return control to op. sys.

/Erase CRT screen

Return control to op. sys. 
The initialization task reads in parameters from the high-speed reader to specify the number of trials, number of digits in the positive and negative sets, the intertrial interval, and the foreperiod before stimulus presentation. The two sets of stimulus digits are then read into tables POSSET and NEGSET. After initializing several other variables, control is transferred directly to task NXTRL. (The GOTO statement at the end of the initialization task could be omitted with the same result.) All tasks end either by transferring control to another task or by returning control to the operating system.

Task NXTRL first requests that the intertrial interval be timed out. It is important to note that execution of the task is not suspended at the TIMEOUT instruction. Instead, processing goes on to the completion of the task, while timing proceeds, at which point control is returned to the operating system. When the interval finally does time out, the operating system turns control over to the task WARNON which was specified in the TIMEOUT instruction. In the remainder of the task, a random number is obtained to determine whether to draw a stimulus digit from the positive set or the negative set, and then another random number is drawn to determine which digit in the chosen set is to be presented. A flag is set if the stimulus digit is from the positive set.

Task WARNON turns on the warning light, requests that the duration of the warning flash be timed out, and requests that the foreperiod be timed out concurrently.

Task WARNOF simply turns off the warning light when the flash duration has timed out.

Task STIMON displays the stimulus digit on the subject's CRT, gets the current time marking the start of the subject's reaction time, and enables the two keys on which the subject responds.

Task RESP has two entry points, one for each of the two possible responses. A flag is set for later reference if the subject indicates a positive set item by his response. The current time is obtained, the CRT screen is erased, and the response keys are disabled so that any further key presses will not cause task execution. If the subject made a correct response, the word "CORRECT" is displayed on the CRT, otherwise "ERROR" is displayed. A request is made to timeout the interval FEEDBK and then to erase the leedback message. The reaction time is then calculated and written onto paper tape, along with the presented digit and the subject's response. Finally, the trial number is incremented and a test made to determine if another trial is to be run.

The sample program requires 244 (decimal) locitions, including storage area for variables and constants, out of approximately 640 locations available to the user. A program to run several subjects simultaneously under the same procedure would require only slightly more space, since only a single copy of each task is needed.

\section{USE OF PEPL IN OTHER LABORATORIES}

The design of the PEPL system allows it to be transported to other laboratories with little modification. The system can be installed in any laboratory having at least the required minimal PDP-8 hardware configuration. It appears possible for a laboratory having more extensive hardware facilities to modify the system to control other devices, keeping in mind the severe constraint of a $4 \mathrm{~K}$ memory. The highly modular design of the operating system makes modification and relocation of routines in menory a relatively easy task.

While the basic system described here may not have all possible features that might be included in a control system, it does offer a considerable amount of power and flexibility given the minimal hardware requirements.

\section{REFERENCES}

KADDEN, R. M. State notation and SKED: A general system for control and recording of behavioral experiments. Behavioral Research Methods \& Instrumentation, 1974, 6, 167-170.

Polson, P. G. SCAT: Design criteria and software. Behavioral Research Methods \& Instrumentation, 1973, 5, 241-244.

Schneider, W., \& Scholz, K. W. Requirements for minicomputer operating systems for human experimentation and an implementation on a 4K PDP-8 computer. Behavioral Research Methods \& Instrumentation, 1973, 5, 173-177.

SternberG, S. High-speed scanning in human memory. Science, $1966,153,652-654$.

Wallsten, T. S. 8TRAN language and the PDP-8 facility at the L. L. Thurstone Psychometric Laboratory, Behavioral Research Methods \& Instrumentation, 1972, 4, 107-108.

Wickens, T. D., Howard, J., Rice, G., \& Millward, R. B. GASPS: A General ASynchronous Processing System for the PDP-8. Behavioral Research Methods \& Instrumentation, 1972, 4, 108-109. 